\title{
Freedom of Live Satellite Transmission and its Effect on State Sovereignty
}

\author{
Abeer Ali Abdalazeez \\ Department of Political Science, College of Law and Political Science, University of Anbar, Iraq \\ abeeralazeez85@uoanbar.edu.iq
}

\begin{abstract}
:
Direct broadcasting through satellite raises a great problem if it is freed the information and programs without limits and restrictions, because it carries risks to the sovereignty of the receiving state. These risks caused by broadcasting programs and information that affect the political, social, cultural, economic, and other risks that befall the receiving country. This led to a conflict between the principle of state sovereignty and the principle of freedom of information flow which is the legal basis for freedom of direct satellite broadcasting and also led to the division of states between a supporter of freedom of direct satellite broadcasting and its primacy over the principle of state sovereignty and opposition to it. This research came to shed light on this problem of an attempt to address and resolve this conflict, by presenting the issue of freedom of direct satellite broadcasting and its impact on the sovereignty of the state within the framework of the rules of public international law to clarify the principles and provisions of international agreements and covenants and decisions related to this topic, as well as a preview of Juristic views and the states' opinion.
\end{abstract}

Keywords: $\quad$ Transmission; Freedom; Sovereignty; Satellite; Live.

Crossref doi https://doi.org/10.51345/.v32i1.185.g201 


\section{حرية البث الفضائي المباشر وأثره على سيادة الدولة$$
\text { عبير علي عبد العزيز }
$$$$
\text { قسم العلوم السياسية، كلية القانون والعلوم السياسية، جامعة الأنبار، العراق }
$$ \\ abeeralazeez85@uoanbar.edu.iq}

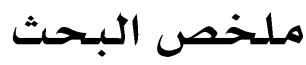

يثير البث الفضائي المباشر عبر الاقمار الصناعية اشكالية كبيرة اذا ما تم اطلاق حرية بثه للمعلومات والبرامج دون حدود وقيود وذلك لما يحمله من مخاطر على سيادة الدولة المستقبلة للبث من خلال بث برامج ومعلومات تؤثر على الجانب السياسي والاجتاعي والثقافي والاقتصادي وغيرها من المخاطر التي تصيب الدولة المستقبلة لمذا البث، مما ادى المى حدوث تعارض بين مبدأ سيادة الدولة وبين مبدأ حرية تدفق المعلومات الذي يعد الاساس القانوني لحرية البث الفضائي المباشر وحدوث انقسام الدول بين مؤيد لحرية البث الفضائي المباشر وتغليبه على مبدأ سيادة الدولة وبين معارض له، لذلك جاء هذا البحث ليسلط الضوء على هذه المثكلة ومحاولة لمعالجة وفك هذا التعارض وذلك من خلال طرح موضوع حرية البث الفضائي المباشر واثره على سيادة الدولة في اطار قواعد القانون الدولي العام لبيان ما جاء في الاتفاقيات والمواثيق والقرارات الدولية من مبادئ واحكام متعلقة بهذا الموضوع، فضلا عن استعر اض الآراء الفقهية وموقف الدول منه.

الكلمات المفتاحية: البث، حرية، السيادة، الفضائي، المباشر.

Crossref doi https://doi.org/10.51345/.v32i1.185.g201 
المقلدمة :

ان التقدم العلمي والتكنولوجي الحاصل في بجال البث الفضائي المباشر كان له الاثر الايجابي الواضح في ختلف الجوانب العلمية والثقافية والاقتصادية والاجتماعية وغيرها وفي نفس الوقت اثار العديد من المشكلات اهمها مشكلة التعارض بين حرية البث الفضائي المباشر وسيادة الدولة على اقليمها؛ وذلك لما يحمله البث المباشر من خخاطر على سيادة الدولة المستقبلة للبث المباشر نتيجة لإمكانيته على بث المعلومات و البرامج أياً كان نوعها متجاوزا بذلك الحدود الاقليمية للدول مما يشكل تهديد لمبدأ السيادة الذي يعد من المبادئ الاساسية التي اكد على احتر امها القانون الدولي لذلك نجد ان الدول انقسمت بين مؤيد لحرية البث الفضائي المباشر وتغليبه على مبدأ سيادة الدولة مستندين على مبدأ دولي اخر وهو مبدأ حرية تدفق المعلومات وبين معارض لحرية البث الفضائي المباشر استنادا المى اهمية احترام مبدأ سيادة الدولة وتغليبه على حرية البث الفضائي المباشر لذلك ارتأينا انه من الضرورة والاهمية البحث في هذه المشكلة مستعرضين بذلك مفهوم البث الفضائي المباشر وعلاقته بمبدأ حرية تدفق المعلومات وما يحمله البث المباشر من خخاطر على سيادة الدولة وذلك كمبحث اول ثم نتناول استعر اض مواقف الدول والآراء الفقهية من التعارض الحاصل بين حرية البث الفضائي المباشر ومبدأ سيادة الدولة خختمين بموقف القانون الدولي من هذا التعارض في ضوء الاتفاقيات والمو اثيق والقرارات الدولية، وذلك في المبحث الثاني وعلى النحو الاتي:

المبـحث الاول: مفهوم البث الفضائي المباشر وعلاقته بهبـأ حرية المعلومات ومخاطره على سيادة الدولة لابد لنا قبل ان الخوض في معرفة العلاقة بين البث الفضائي المباشر ومبدأ حرية المعلومات ان نتطرق الى مفهوم البث الفضائي المباشر وتحديد المقصود منه ومن ثم التطرق الى العلاقة بينه وبين مبدأ حرية المعلومات بعده اساساً قانونياً للبث الفضائي المباشر ومن بعدها نستعرض اهم مخاطر البث الفضائي المباشر على سيادة الدولة وسيكون ذلك على مطلبين وعلى النحو الاتي: 
المطلب الاول: مفهوم البث الفضائي المباشر وعلاقته بهبـدأ حريـة المعلومات الفرع الاول: مفهوم البث الفضائي المباشر يمكن تعريف البث الفضائي المباشر بأنه: "المعلومات والاخبار التي تصدر عبر الاقهار الصناعية وتوجه مباشرة الى خختلف دول العالم دون تدخل من الدول ودون ادنى سيطرة حكومية ويستوي في ذلك البث التلفزيوني والاذاعي"(1) وعرفه الاتحاد الدولي للاتصالات بأنه: "خدمة اتصالات بالراديو يتم استقبال الاشارات الصادرة من محطة فضائية موجودة على قمر صناعي ذو مدار ثابت من قبل افراد الجمهور مباشرة"(2) كما ورد تعريف البث الفضائي المباشر من قبل المشرع العراقي في قانون شبكة الاعلام العراقي رقم 26 لعام 2015 مشتملا على البث المباشر وغير المباشر فعرفته (م4/ف1) بأنه: "بث المعلومات او البرامج عبر الاتصالات التلفزيونية او الراديوية او أية وسيلة اخرى لإستقبالها من الجمهور مباشرة أو بواسطة محطات إعادة البث فضائية كانت او ارضية وبشكل مجاني"(3). إذن فالبث الفضائي المباشر وبحسب التعريفات السابقة يتضمن البث التلفزيوني المباشر والبث الاذاعي المباشر، مع الاخذ بعين الاعتبار الاهمية الكبيرة التي يهظى بها البث التلفزيوني المباشر كونه يجمع بين الصوت و الصورة مما يجعل تأثيره واقناعه للمستقبل أكثر من غيرها من وسائل الاقناع(4). وتجدر الاشارة الم ان البث الفضائي المباشر يختلف عن البث الفضائي غير المباشر في ان الاول يكون بأرسال القمر الصناعي اشارات الكترونية قوية تحمل عبرها البرامج من محطات الارسال الى الجمهور مباشرة لاسيا في المنازل عن طريق اجهزة التلفزيون والتي تكون بجهزة بهوائي خاص يسمى(Dish)(5)، أما البث الفضائي غير المباشر فيكون أيضا عبر إرسال الاقمار الصناعية لإشارات حاملة عبرها البرامج، إلا انها تكون عاجزة عن الوصول المباشر الم الجمهور الا بتدخل عحطة استقبال ارضية تقوم بدور الوسيط لاستقبال هذه الاشارات ومن ثم خضوعها لعمليات تقنية للتحول الى ارسال مقروء من الجمهور بواسطة اجهزة الاستقبال العادية (التلفزيون) فيتم الارسال اليها من محطات ارضية(6)، فمعيار التمييز بين النوعين بأن الاول لا يحتاج الى محطة ارضية لإستقبال 
اشارات القمر الصناعية وبثها لجمهور الناس، اما النوع الثاني فأنه يستلزم وجود محطة ارضية تستقبل اشارات القمر الصناعي ثم تعيد بثها لجمهور الناس، وألامر ينطبق على البث الاذاعي ايضا(7). وبالتالي فأن البث الفضائي المباشر يعتمد على وجود ثلاث عناصر هي: 1 - القناة التلفزيونية التي تبث البرامج او الحدث. 2- القمر الصناعي الذي يلتقط البث ويعيد بثه مباشرة لجمهور الناس. 3- المتلقي وهو جهاز التلفزيون العادي مزود بجهاز التقاط خخصص للبث التلفزيوني المباشر، اذيستقبل ما يبثه مباشرة دون وسيط (8). الفرع الثاني: العلاقة بين البث الفضائي المباشر ومبدأ حرية المعلومات (حرية المعلومات كأساس قانوني للبث الفضائي المباشر ) يرتبط البث الفضائي المباشر لاسيا البث التلفزيوني ارتباط وثيق مع مبدأ "حرية المعلومات" بل ان مبدأ حرية المعلومات يعد الاساس القانوني للبث الفضائي المباشر، والمقصود بحرية المعلومات هو "الحق في صناعة المعلومات والوصول الى مصادرها بالإضافة الم تعدد تلك المصادر"(9)، وقد تم التأكيد على هذا المبدأ في العديد من المواثيق الدولية، اذ ورد مبدأ حرية تداول المعلومات في الجمعية العامة للأمم المتحدة بموجب القرار 59/ 1 لعام 1946 والذي نص على ان "حرية المعلومات حق اساسي من حقوق الانسان، وانها المحك لكل الحريات التي تهتم بها الامم المتحدة وتدافع عنها ... ومعيار كافة الحقوق والحريات التي من اجلها تم تكريس الامم المتحدة"(10) كما ورد هذا المبدأ في (م919) من الاعلان العالمي لحقوق الانسان عام 1948 فنصت على ان "لكل شخص الحق في حرية الرأي والتعبير ويشمل هذا الحق حرية اعتناق الآراء دون تقييد بالحدود الجغر افية"(11). وبالتالي نجد ان حق حرية المعلومات يتضمن ثلاث عناصر وهي: 1 - حرية صناعة المعلومات ونشرها والاتصال بالآخرين. 
2- حق الانسان في الخصول على المعلومات والسعي اليها متمثلة في بجموعة الانشطة التي تؤدي الى الحصول على المعلومة والمعرفة من دون معوقات.

3- حق الانسان في تلقي المعلومات واستقبال وتلقي الافكار والآراء لزيادة قدرته الثقافية والاجتماعية والسياسية من مصادر متعددة ومستقلة (12). ومن هنا نستخلص بأن البث الفضائي المباشر عبر الاقمار الصناعية له صلة وثيقة بحرية المعلومات والحق في الاتصال، فعبر البث الفضائي المباشر تنتقل الافكار والمعتقدات المى دول اخرى، كما يعد وسيلة لمارسة الحق في الاتصال بين الدول، فالبث التلفزيوني المباشر له صلة وثيقة ومباشرة في حرية المعلومات وتداولها كونه يقبل حرية المعلومات في صورتها المطلقة التي لا تتقيد بالحدود السياسية ولا بحا تفرضه الانظمة الداخلية من حدود(13). لذلك فأن التأكيد على مبدأ حرية المعلومات وحق الانسان في الحصول عليها قد اوحى بعدم خضوع البث المباشر بالأقمار الصناعية لأية قيود لاسيا القيد الخاص بأخذ الاذن المسبق من قبل الدولة بالبث المباشر وذلك مراعاة لإعتبارات حرية تداول المعلومات وحق الانسان في الحصول عليها مما يقودنا لنتيجة وهي عدم فرض أي نوع من القيود والالتزامات على البث المباشر، الا ان هذه النتيجة غير منطقية لتعارضها مع مبدأ دولي اخر وهو سيادة الدول على اقليمها(14) وهذا ما سنتطرق لتوضيحه بصورة تفصيلية في المبحث الثاني.

المطلب الثاني: مخاطر البث الفضائي المباشر على سيادة الدولة لاشك ان للبث المباشر عبر الاقمار الصناعية العديد من المزايا والاثر الايجابي على الدول، الا انه له سلبياته وخاطره على الدول في جوانب عدة، فعلى الرغم من ان للبث المباشر دور ايجابي في ججال نشر التربية والتعليم والذي تجسد في الاتفاقية الخاصة بالبث السمعي المرئي الثقافي المبرمة بين الولايات المتحدة و الهند لعام 1969 والتي تبلورت بشكل بث برامج تلفزيونية تعليمية مباشرة الى الهند عام 1975 وذلك لان البث التلفزيوني المباشر بالأقمار الصناعية يعد من اهم الوسائل التي تخدم الجانب التعليمي نتيجة لما يتميز به من سهولة تغطيته لإماكن بعيدة وشاسعة وو صوله بتكاليف زهيدة لاسيا للدول النامية(15) بالإضافة الم انه فتح افاق واسعة من 
المعرفة والاطلاع عن قرب على العالم متجاوز كل الحدود بحيث يمكن المشهد من الحصول على اهم الاحداث

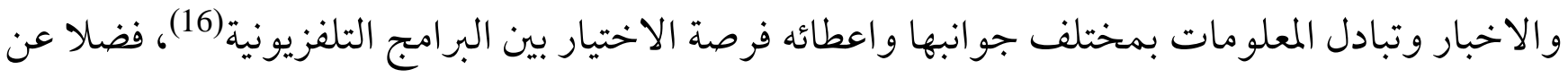
قدرته على اجتياز العوائق الطبيعية كالجبال والمحيطات وعدم مواجهة عقبات التشويش وتوفير استقبال الخدمة بجودة ودقة عالية(17)، الا انه الى جانب هذه الايمابيات التي يهققها البث الفضائي المباشر سلبيات وخاطر كثيرة لاسيا على سيادة الدولة، فمبدأ سيادة الدولة يمنح للدولة "سلطة عليا على رعاياها واستقلالها عن اية سلطة اجنبية وينتج عن هذا ان يكون للدولة كامل الحرية في تنظيم سلطاتها التشريعية والادارية

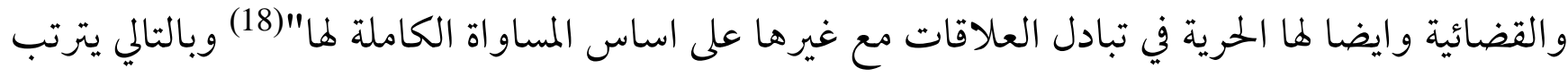
على مبدأ سيادة الدولة آثار عدة اهمها، المساواة بين الدول وعدم جواز التدخل في شؤون الدول الاخرى وحرمة الاقليم وسلامته وحرية اختيار نظام الحكم المناسب لظروفها وغيرها من الآثار (19)، فوفقاً لهذا المبدأ يجق لكل دولة ان تختار نظامها السياسي والاجتماعي والاقتصادي من دون أي تدخل وتأثير خارجي لأي

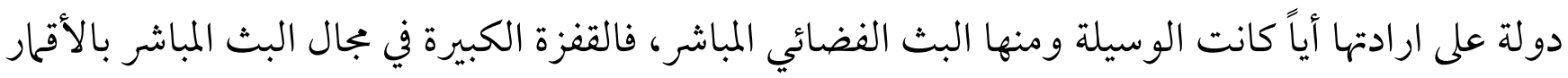
الصناعية زاد من تهديد وانتهاك سيادة الدولة من خلال ما يبثه من معلومات تؤدي المى خلق تفاعل من الجانب النظري والعملي بين سيادة الدولة وحرية البث الفضائي للمعلومات المتدفقة نتيجة للدور المؤثر للمعلومات الذي يؤدي الى تغليب احدى الثقافات او النظم الاجتماعية على غيرها من الثقافات نتيجة لما تملكه تلك الثقافة من وسائل اتصال مباشر تساعدها في تدفق وغزو الثقافات الاخرى وطمسها (20). لذا كان من نتيجة ذلك تزايد خخاطر البث الفضائي المباشر على سيادة الدولة الوطنية ومن جملة هذه المخاطر 1- يعد البث الفضائي المباشر بالأقار الصناعية من اكثر الوسائل الاعلامية خطورة في ججال السياسة الدولية، بل يعد الوجه الاخر للإستعمار العسكري من قبل الدول المتقدمة في الوقت الحاضر لما تملكه من

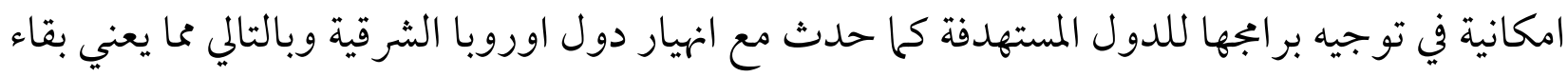


الهيمنة للدول المتقدمة(21)، لذا يرى بعض الفقهاء بأن الدول اليوم تواجه اليوم صورة من صور الحروب الحديثة وشكلا جديدا من اشكال العدوان يسمى "العدوان الاذاعي"(22). 2- طغيان قيم وعادات وثقافات لمجتمعات دول مختلفة -كالمجتمعات الغربية- من حيث الشكل والمضمون عن بجتمعات دول اخرى، فالبث الفضائي المباشر بها يبثه من معلومات متجاوزا الحدود الجغر افية للدول يؤدي الى تجاوز سيادة الدول من خلال المس بخصو صياتها الثقافية، فالسيادة ليست سلطة فقط بل هي سيطرة الدول على معلوماتها لذلك فقد اخذت هذه السلطة تضعف تدريجيا امام ظهور تنميط متزايد للسلوك البشري في اتجاه ما يسمى (أمركة الثقافة)(23)، فالبث المباشر عبر الاقمار الصناعية قد يضعف الجانب الحضاري ويقلل من قدرته في مو اجهة المستحدث و الو افد الذي يهدد حرية الدولة او كفاءتها الانتاجية او ملئ فراغها الوجداني والعقلي بأفكار دخيلة لا تمت الى اصالتها بقدر ما تمت المى المصادر الوافدة او الغازية خاصة اذا كان هذا الجلديد براقا ومبهر|(24) محا ترك اثره السلبي على التشئة الاجتماعية وتغير القيم والسلوك الاجتماعي والاخلاقي لمجتمع الدولة المستقبلة للبث من خلال الترويج لثقافات معينة كالترويج للثقافة الاباحية وغيرها من الثقافات والسلو كيات المخالفة لسلوكيات ومبادئ وقيم المجتمع المستقبل للبث(25). 3 - كما تكمن خطورة البث المباشر عبر الاقمار الصناعية لاسيا (البث الغربي) في إنه يعد اداة لتوجيه الرأي العام العالمي كونه يعد من المصادر الاساسية الموثوقة للمعلومات للعديد من الدول لاسيا الدول العربية، فترى الشعوب من خلالها الاحداث و الوقائع العالمية بالرغم من ان مؤسسات البث الفضائي المباشر هذه عادة ما تعبر عن السياسة الخارجية للدولة التي تنتمي اليها وتخدم مصالحها ومصالح مالكيها (26) 4- يعد البث المباشر في بعض الحالات تدخل في الشؤون الداخلية للدولة المستقبلة للبث من خلال قيام الدولة الباثة بالترويج والدعاية لمعتقدات سياسية خخالفة لسياسة الدولة المستقبلة(27)، او تشوية الاخبار بها يخدم مصالحها السياسية والاقتصادية وتركيزها على المعلومات والاخبار المتعلقة بالانقلابات 
العسكري والتدهور الاقتصادي في الدولة المستقبلة من اجل تحقيق عدم توازن في بث المعلومات من

$$
\text { اجل ضمان بقائها في دور المسيطر المتحكم في المعلومات التي تبث للدول الاخرى. }
$$

5- ان قدرة فضائيات البث المباشر من التأثير في الفرد والجماعة جعلها تستغل وسيلة البث المباشر في

الترويج للسلع والمنتجات الاجنبية واغراق اسواق الدول النامية بالسلع والمنتجات الغربية مما يشكل

$$
\text { خطر اعلى اقتصادها (28). }
$$

اذن فالبث الفضائي المباشر سواء كان السمعي او البصري يمكن ان يتحول المى اسلحة خطيرة تدعو الى الحروب والاعتداء واشعال الفتن وتغيير ثقافات وقيم وسلوكيات بجتمعات الدول المختلفة وفرض ثقافات وتقاليد المالكة لوسائل البث الفضائي المباشر مما ادى الى مناداة الفقه الدولي بضرورة وضع حلول للتصدي لمخاطر البث الفضائي المباشر وتوسيع مفهوم السيادة والذي نتج عنه ظهور فكرة السيادة المعلوماتية والثقافية(29) والتي سنتناوها في المبحث الثاني.

\section{المبـحث الثاني: موقف القانون الدولي من التعارض بين البث الفضـائي المباشر وسيـادة

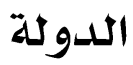

بعد ان استعرضنا في المبحث الاول مفهوم البث الفضائي المباشر وارتباطه بمبدأ حرية تداول المعلومات وما يحمله من غخاطر على سيادة الدول نجد ان المواقف الدولية والآراء الفقهية قد انقسمت بين مؤيد لإطلاق حرية البث الفضائي المباشر وتغليبه على مبدأ سيادة الدولة وبين مقيد للبث الفضائي المباشر في تدفقه للمعلومات احتراما لمبدأ سيادة الدولة وعدم انتهاكها، لذاك نرى من الاهمية ان نبين الموقف الدولي -الفقهي والقانوني - من التعارض بين حرية البث الفضائي المباشر للمعلومات وبين سيادة الدولة وسيكون على النحو 


\section{المطلب الاول: موقف الفقه الدولي من التعارض بين حريـة البـث الفضـائي المباشـر وسـيادة}

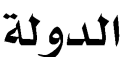

الفرع الاول: الفقه الدولي الذي يغلب حرية البث الفضائي المباشر على مبدأ سيادة الدولة:

وينادي بهذا الرأي اغلب فقهاء الدول الغربية المتقدمة إعلاميا ويذهبون بإتجاه تغليب حرية البث الفضائي المباشر عبر الاقمار الصناعية على مبدأ سيادة الدولة حماية وضمان لمبدأ حرية المعلومات(30)، ويؤكد على ضرورة ان تكون حرية بث المعلومات مطلقة وعدم تقييدها بأية قيود لاسيا القيد المتعلق بالحصول على التصريح المسبق بالبث الفضائي المباشر ماعدا القيود المتعلقة بالقواعد القانونية لحماية النظام العام(31)، لذلك فأن الدولة ملزمة بإتاحة تدفق المعلومات عبر الفضائي المباشر بالأقمار الصناعية من دون اية قيود حتى وان كان هذا التدفق قد يؤدي المى التعارض مع سيادة هذه الدولة او غيرها(32)، ويبرر الفقه الدولي رأيه هذا بأن الحق بالبث والاتصال والتدفق الحر للمعلومات من حقوق الانسان الاساسية التي اكدت عليها العديد من المواثيق و القرارات الدولية -تم ذكرها سابقا- ومن باب اولى عدم جواز ايقاف البث الفضائي المباشر لأي دولة او تقييد البث الحر للمعلومات وبالتالي لا توجد ضرورة لوضع قواعد قانونية تنظم انشطة البث المباشر (33)، كما ان تلقي المعلومات عبر البث المباشر بالأقمار الصناعية يعد من الضمانات الاساسية في حصول الفرد على حقه في تلقي المعلومات وتعدد مصادرها واستقلاليتها وضمان صحتها ومنع احتكار وسيطرة الدولة لوسائل المعلومات الذي يهدد حق الفرد في حرية التعبير وتكوين آرائه ومعتقداته الخاصة به، وبالتالي فأن البث الحر المباشر بالأقمار الصناعية من وجهة نظر هذا الفريق يزيد من قدرة الفرد الثقافية والاجتماعية والسياسية(34)، فضلا عن ان اصحاب هذا الرأي يبررون موقفهم بإدعائهم ان حرية البث المباشر في تدفقه للمعلومات بدون قيود سيساعد الدول النامية في حصوها على المعلومات التي تتناسب مع اوضاعها محا يؤدي الى نهوضها، وبالتالي فأن حرية البث المباشر يعد بمثابة مساهمة من الدول المتقدمة في مساعدة وتمكين الدول النامية في الحصول على الاختيار المتوازن للمعرفة بدلاً من دفعها لمزيد من الفقر أو الموت جوعاً، بالإضافة المى انهم يرون بأن الحصول على المعلومات عبر الاقمار الصناعية يرتبط بصورة اساسية بإستهلاك المعلومة المعروضة من قبل 
الدولة الباثة يشبه استهالك المعلومات التي يمكن الوصول اليها من خلال المكاتب والوثائق والمعارض وغيرها، فالفرد هنا لا يفعل شئ سوى استقبال المعلومة المعدة للإستهالك وتخزينها كما يفعل البث المباشر عبر الاقمار الصناعية ومن ثم من واجب الدولة ان تلتزم بتمكين الفرد في حصوله على المعلومات بدون أي عراقيل او قيود(35)، وتجدر الاشارة الى ان من اهم الدول التي تزعمت هذا الرأي وغلبت مبدأ حرية البث الفضائي هي الولايات المتحدة الامريكية وبريطانيا(36)، الا انه بالرغم من ان الولايات المتحدة الامريكية قد تزعمت الفقه الغربي وانحازت بصورة مطلقة المى حرية البث والتدفق الحر للمعلومات عبر الاقمار الصناعية من دون اي قيود نجدها قد اوردت بعض القيود في قوانينها الداخلية على البث الفضائي المباشر المتعلقة بحماية حقوق المؤلف والبث الاباحي ومنع القذف والتشهير وعدم نشر المعلومات والوثائق السرية ومنع الاعلانات المزيفة وغيرها من المحظورات بينما رفضت في الوقت نفسه ان تخضع حرية المعلو مات عبر البث المباشر لأي قيود على الصعيد الدولي (37) كما يبررون موقفهم بأن الدول لم تبدي اعتراضها على البث الاذاعي الموجه اليها منذ بداية ممارسته، فلم تشرع أي قو انين تقيد وتمنع موطنيها من سماع الاذاعات المعادية وغيرها(38).

الفرع الثاني: موقف الفقه الدولي الذي يغلب مبدأ سيادة الدولة على حرية البث الفضائي المباشر: ويذهب بهذا الإتجاه اغلب الدول النامية والفقه العربي والاتحاد السوفيتي سابقا والذي يغلب مبدأ سيادة الدولة على حرية البث الفضائي المباشر وتدفق المعلومات(39)، وذلك لما يشكله حرية بث المعلومات من انتهاك لحقوق الدولة الموجه البث اليها واعتداء على سيادتها الاقليمية مما يؤثر على هويتها الثقافية والسياسية والاجتماعية(40) فضلا عن ان مبدأ سيادة الدولة يعد من المبادئ الاساسية في القانون الدولي ويكظى بقبول المجتمع الدولي، فسيادة الدولة يعني حق الدولة في الاستقلال السياسي وتقرير المصير واساس العلاقات بين الدول القائمة على الاحترام المتبادل لحقوق الدولة السيادية وعدم التدخل في الشؤون الداخلية للدول(41)، وما البث المباشر بالأقمار الصناعية الا احد خدمات الاتصالات التي تعد من اختصاصات الدولة التي تنظمها وحدها استناداً المى القاعدة القانونية الدولية التي تنص على حق الدولة السيادي في تنظيم اتصالاتها وبالتالي 
اكتسابها لصفة الدولية والمحلية في نفس الوقت مما يعني خضوع البث المباشر الموجه المى الدولة المستقبلة لسيادة الدولة المستقبلة كونه يدخل ضمن خدمات الاتصالات(42)، لذلك تستند الدول النامية الفقيرة في تأييدها لتقييد حرية البث المباشر على تمسكها الظاهري والقولي بالسيادة المطلقة على اقليمها وانعدام قدرتها في تملكها لتقنية منع تجاوز البث المباشر والتشويش عليه او ايقافه لمنع تأثيره الضار على مو اطنيها(43)، كما ان في الوقت الذي يعد مبدأ سيادة الدولة من المبادئ العرفية الرئيسية في القانون الدولي فأن مبدأ حرية المعلومات وتدفقها الحر لا يعد كذلك وانما هناك اختلاف شديد بين الدول من حيث ممارستها لهذا المبدأ، اذ نجد ان بعض اراء الفقهاء ذهبت المى القول بأن حرية المعلومات ليست مبدأ قانوني(44)، فبالرغم من النص على حرية الرأي والمعلومات في (م19 ) من الاعلان العالمي لحقوق الانسان وفي (م19 و02 ) من العهد الدولي للحقوق المدنية والسياسية لعام 1966 الان ان البعض يراه مبدأ آيديولوجي فقط ولا قيمة الزامية له عند الدول(45). لذلك نجد ان احد الفقهاء المناصرين لإتجاه تغليب سيادة الدولة على حرية بث المعلومات يرى بأن العديد من الدول الغربية تأخذ بالتفسير الواسع لمعنى حرية المعلومات وتطمح ان يكون البث المباشر عبر الاقمار الصناعية غير مشروط بالمو افقة المسبقة من قبل الدولة المستقبلة، الا اننا نرفض ذلك من اجل ان يسود السلام وضماناً للحفاظ على سيادة الدولة وعدم التدخل في الشؤون الداخلية وحماية الهوية الثقافية لكل دولة(46). وبالتالي فأن التفاف بجموعة من الدول حول مفهوم حرية البث بغض النظر عن تعارضه مع سيادة الدولة من عدمه لا يعني بالضرورة الزام الدول كافة بتطبيق ذلك المبدأ ، فهناك بعض الدول كفرنسا والسويد والنرويج نجد انها قيدت من حرية بث المعلومات واكدت على خضوع البث المباشر عبر الاقمار الصناعية لقواعد القانون الدولي بها فيها مبدأ سيادة الدولة ومبدأ عدم التدخل في الشؤون الداخلية، واهتمت بوضع قواعد وضو ابط قانونية داخلية تنظم البث المباشر وتدفق المعلومات بالإضافة المى وضع تقنين لقواعد حسن السلوك التي تحكم انشطة البث التلفزيوني المباشر وتحريم كافة انواع البث المباشر الضارة(47). 


\section{المطلـب الثـاني: موقـف القـانون الــدولي مـن التعــارض بــين سـيـادة الدولــة وحريــة البـث} الفضائي المباشر

ان مبدأ سيادة الدولة ومبدأ حرية البث وتدفق المعلومات قد تضمنتها العديد من الاتفاقيات والمو اثيق الدولية محا ادى المى انقسام دولي في تغليب احد المبدأين على الاخر، لذلك سنبين في هذا المطلب موقف القانون الدولي من خلال الاتفاقيات والقرار ات الدولية وعلى النحو الاتي: لقد ذكرنا سابقا بأن مبدأ حرية بث المعلومات قد ورد في العديد من المعاهدات والمواثيق الدولية، ففي الوقت الذي اكد فيه العهد الدولي للحقوق المدنية والسياسية في (م 29) لعام 1966 على حق كل شخص في تلقي المعلومات ونشرها الا انه كذلك اكدت في (ف3 ) من نفس المادة على منح الدولة سلطة وضع القيود على ممارسة هذا الحق اذا كان ذلك ضروريا لحماية سيادة الدولة وحماية النظام العام وغيرها(48)، مما يعني ان العهد الدولي للحقوق المدنية والسياسية قد غلب سيادة الدولة على حرية بث المعلومات في حالة التعارض فيها بينها، اضافة الى انه بالرغم من وجود قاعدة قانونية دولية تضمنتها اتفاقية الاستخدام السلمي للبث الاذاعي المبرمة في جنيف لعام 1936 تقضي بحرية البث الاذاعي الموجه الم الدول الاخرى الا انه اوردت على هذه القاعدة استثناءات عديدة اهمها تحريم الدعاية الهدامة والعدائية الموجهة للإخلال بأمن الدولة واستقلالها، وتحريم محطات القرصنة التي تقوم بأنشطة البث من خارج اقليم الدولة المستقبلة ومن دون ترخيص من دولة الاصل، وتحريم البث المؤدي المى تداخلات ضارة مع خدمات وانشطة البث التي تقوم بها الدول الاخرى بالإضافة الى ه تحذير الدولة الصادر منها بث غير شرعي من خطورة فعلها وضرورة التوقف عنه(49)، وتجدر الاشارة الم انه في عام 1972 تمت الموافقة في منظمة اليونسكو على اصدار اعلان يتضمن المبادئ التي تحكم نشاط البث الاذاعي عبر الاقمار الصناعية وكان من اهم مبادئها ما ورد في (م1 ) من الاعلان وهو ان قواعد القانون الدولي ومعاهدة الفضاء الخارجي وميثاق الامم المتحدة تنطبق على البث الاذاعي عبر الاقمار الصناعية وعلى جميع الدول احترام مبدأي السيادة والمساواة بين اعضاء الاسرة الدولية، لذلك عد هذا المبدأ نقطة تحول مهمة بدأت الدول من خلالها الربط بين حرية البث والتدفق المعلوماتي وبين ضرورة احترام سيادة الدولة المستقبلة وبالتالي 
فأن كل دولة تبث برابجها عبر الاقمار الصناعية وبصورة مباشرة عليها مراعاة القوانين المحلية للدول المستقبلة للبث(50)، اما بالنسبة للأهم القرارات الدولية المتعلقة بهذا الشأن هو قرار الامم المتحدة رقم (92 / 7 ) لعام 1982 الخاص بالمبادئ التي تحكم استخدام الدول للأقمار الصناعية في البث التلفزيوني المباشر والذي جاء مؤكداً على ضرورة الحصول على المو افقة المستقبلة للبث المباشر (51) اذ تضمنت ف(ز) من المبدأ السابع على ان كل دولة ترغب في اقامة خدمة البث المباشر الدولي من اقليمها، ان تخطر على وجه السرعة رغبتها المى الدولة او الدول المستقبلة وان تدخل في مشاورات مع كل دولة تطلب ذلك(52). ونستتج من ذلك ان القانون الدولي يفرض قيدا اخرا على حرية البث الفضائي المباشر وهو ضرورة حصول الدولة الباثّة على الموافقة المسبقة من قبل الدولة المستقبلة للبث بالإضافة المى ان المبدأ الاول من القرار المذكور جاء مؤكداً على انه يستلزم ان يكون الهدف من البث الفضائي المباشر التنمية الشاملة في المجالات الاقتصادية والاجتحاعية وغيرها مع ضرورة مراعاة الحقوق السيادية للدولة وعدم التدخل في الشؤون الداخلية للدولة)(53). إذن ومن خلال ما تم عرضه يتبين لنا ان الاصل سيادة الدولة والاستثناء هو حرية البث، وان مصير سيادة الدولة امام التدفق الكبير في المعلومات على اقليمها دون رقابة قد اوضحته الجمعية العامة للأُمم المتحدة من فئن خلال قرارها رقم (3268/298 29 مؤكدة على انه لا يجب استخدام التطورات العلمية والتقنية بشكل مخالف لمبادئ القانون الدولي الاساسية وعلى رأسها مبدأ سيادة الدولة وبذلك تكون قد وضعت قيدا على جميع الدول

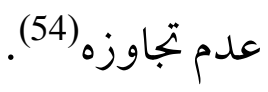

ويترتب على ذلك ان حرية تدفق المعلومات عبر البث الفضائي المباشر يجب ان يارس بشكل متوازن، بمعنى ان يكون بث المعلومات وفقا لما يتضمنه النظام الدولي الجديد للمعلومات ( The New World Information (Order والاخلاقية والثقافية، لذا نجد ان بعض الدول اخذت بفكرة التدفق المتوازن للمعلومات في بجال البث

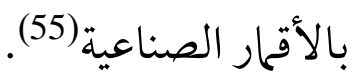


اخيراً ويعد ان استعرضنا موقف الفقه والقانون الدولي من التعارض بين حرية البث الفضائي وسيادة الدولة نجد انه يجب ان تكون هناك موازنة بين البث المباشر عبر الاقمار الصناعية ومبدأ سيادة الدولة، اذ يمكن للأقمار الصناعية ان تمارس نشاطها ببث وتدفق المعلو مات اذا ما كان في اطار الشرعية الدولية وبالشكل الذي لا يمس بالمصالح الاساسية للدول(56)، ويتحقق ذلك من خلال احترام المبادئ الدولية الاساسية التي نظمت نشاط الاقمار الصناعية والتي من اهمها مبدأ الاستخدام السلمي للفضاء الخارجي الواردة في معاهدة الفضاء الخارجي لعام 1967 ومبدأ التعاون الدولي في الانشطة الفضائية الواردة في قرار الجمعية العامة للأمم المتحدة الخاصة بالتعاون الدولي لعام 1959 ومبدأ التراث المشترك للإنسانية الواردة في معاهدة الفضاء الخارجي وغيرها من القوانين والمواثيق والقرارات الخاصة بالاتصالات و البث عبر الاقمار الصناعية.

بعد اكملنا بحثنا حول حرية البث الفضائي المباشر واثره على سيادة الدولة توصلنا الى النتائج الاتية: 1 - بعد تناولنا لمفهوم البث الفضائي المباشر تبين لنا بأن خطورته تكمن في بثه للبرامج والمعلومات عبر ارسال القمر الصناعي لإشارات تصل مباشرة للجمهور عبر اجهزة التلفاز المزودة بهوائي (Dish) وهي بذلك تختلف عن البث الفضائي غير المباشر الذي يكون بأرسال اشارات من القمر الصناعي الا انها لاتصل مباشرة للجمهور الا بتدخل محطة استقبال ارضية تقوم بتحويلها للجمهور وبالتالي انعدام تهديدها لسيادة الدولة.

2- توصلنا من خلال استعراض العلاقة بين البث الفضائي المباشر ومبدأ حرية المعلومات الى ان هناك علاقة وثيقة ومترابطة بين الاثنين بل ان حرية المعلومات يعد الاساس القانوني في البث الفضائي المباشر وتدفقه للمعلو مات بعده من المبادئ التي تم التأكيد عليها في العديد من المواثيق والقرارات الدولية. 3- من خلال بيان مخاطر حرية البث الفضائي المباشر على سيادة الدولة توصلنا الى ان المخاطر تتنوع بتنوع البرامج والمعلومات التي يتم بثها فهناك خخاطر ذات طبيعة سياسية وذهب البعض الى عدها الوجه 
الاخر للاستعمار العسكري وهناك مخاطر ذات طبيعة اجتماعية وثقافية تهدد قيم وعادات وثقافة المجتمع في الدولة المستقبلة للبث، وهناك خخاطر ذات طبيعة اقتصادية يكون من خلال الترويج للسلع والمنتجات الاجنبة للدولة الباثة واغراق اسواق الدول المستقبلة لاسيا الدول النامية بهذه السلع و المنتجات، فضلا عن ان خطورته تكمن في التأثير على الرأي العام العالمي من خلال بث برامج موجهة بما يخدم مصالح الدولة او المؤسسة الباثّة، واخيرا يخاطر البث الفضائي التي تشكل تدخل في الشؤون الداخلية للدولة المستقبلة لهذا البث. 4- توصلنا من خلال استعراضنا لموقف الفقه والقانون الدولي من التعارض بين حرية البث الفضائي المباشر وسيادة الدولة المى وجود انقسام بالر أي ، فهناك من يغلب حرية البث الفضائي المباشر على سيادة الدولة مستندا بذلك بعلد من الحجج المتضمنة لمواثيق وقرارات دولية اكدت على حرية البث الفضائي وتدفق المعلومات ،وهناك من يغلب سيادة الدولة على حرية البث الفضائي مقدما ايضا حججه ومبرراته المستندة على مواثيق وقرارات دولية ،ومن ثم خلصنا الى نتيجة مفادها بأنه سيادة الدولة هو الاصل وان الاستثاء هو حرية البث الفضائي المباشر.

1 - ان يتم استخدام البث الفضائي المباشر بشكل متوازن وبها لا يشكل خرق لقواعد القانون الدولي ومبادئه الاساسية، بمعنى ان حرية البث الفضائي يجب ان تمارس بالطريقة التي لا تمس سيادة الدولة وقيمها الاجتماعية والثقافية والسياسية وغيرها، ويتحقق ذلك من خلال التزام الدولة الباثّة بالقيود والمبادئ التي تضعها الاتفاقيات الدولية الجماعية والثنائية بين الدولة الباثة والمستقبلة والقرارات الدولية فضلا عن القوانين الداخلية للدولة المستقبلة وعلى رأسها القيد المتضمن وجوب اخذ الموافقة المسبقة من الدولة المستقبلة للبث الفضائي المباشر. 
2 - ضرورة توحيد جهود دولية مشتركة للدول لاسيا الدول النامية والمتقدمة لعقد اتفاقية موحدة تنظم نشاط البث الفضائي المباشر من اجل تلافي التفاوت في الامكانية والقضاء على الصراعات الناجمة بينها نتيجة للتعارض الحاصل بين مبدأ حرية المعلومات وتدفقها وبين مبدأ سيادة الدولة وايياد موائمة وموازنة بين الامرين فضلا عن معالجتها لكل ما يستحدث من جوانب متعلقة بالبث الفضائي المباشر وبالشكل الذي ينسجم مع المتغيرات التي تطر أ على مفهوم السيادة في الوقت الحاضر.

\section{المصادر والمراجع: المبل}

1. بن ممودة، ليلى، الاستخدام السلمي للفضاء الخارجي، المؤسسة الجامعية للدراسات والنشر والتوزيع، ط1،

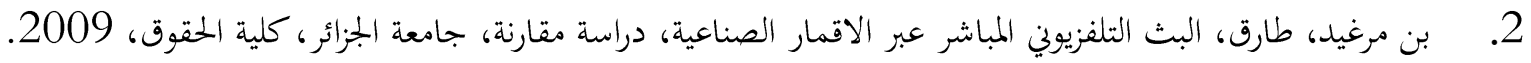

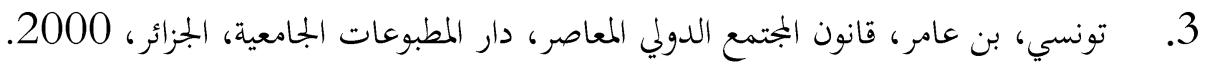

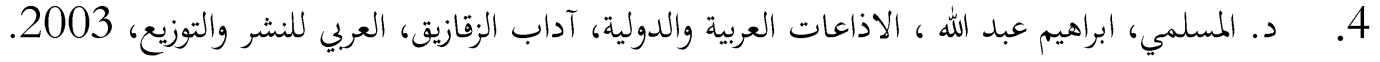

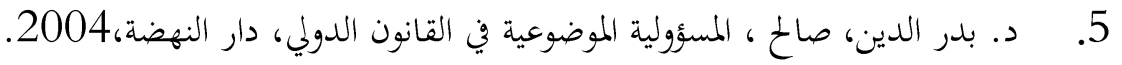

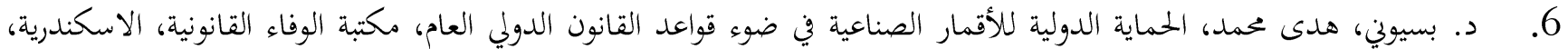

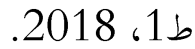
7. د. جمال الدين، صلاح الدين ، البث التلفزيوني المباشر للأقمار الصناعية ،دار النهضة العربية، 1997. 8. د. زناتي، عصام ، التلفزيون المباشر عبر الاقمار الصناعية، دار النهضة العببية، القاهرة، 1991.

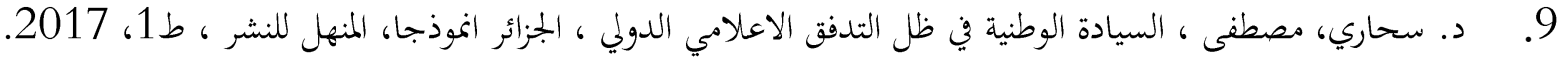

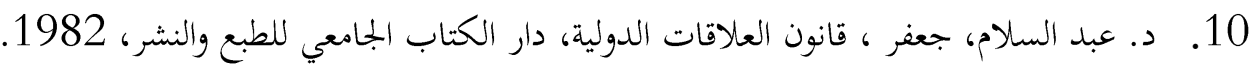

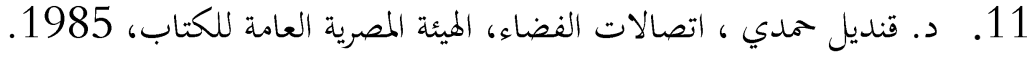
12. د. محمود، محمود حجازي ، النظام القانوني الدولي للاتصالات بالاقمار الصناعية، دار النهضة العربية ، القاهرة، 2001.

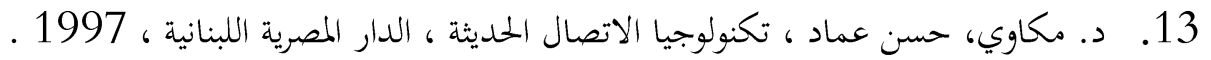
14. لطفي، محمد حسام، البث الاذاعي عبر التوابع الصناعية وحقوق المؤلف، دراسة في العلاقة بين الفضائيات العربية والملكية الادبية

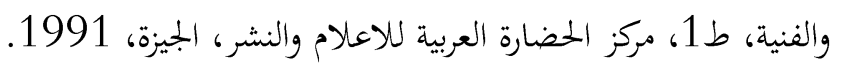

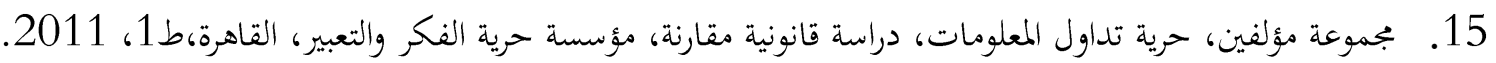

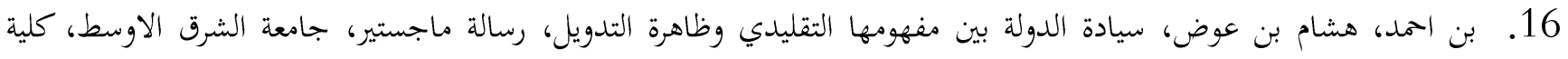
الحقوق، 2013.

17. د. يونس، حممد مصطفى ، النظرية العامة لعدم التدخل في شؤون الدول، رسالة دكتوراه، كلية الحقوق، جامعة القاهرة 1985. 
18. سيد، أحمد فوزي عبد المنعم، المسؤولية الدولية عن البث الاذاعي عبر الاقمار الصناعية في ضوء احكام القانون الدولي العام، رسالة دكتوراه، جامعة القاهرة، كلية الحقوق ،2002.

19. عثمان، جمال عبد الفتاح ، المسؤولية الدولية عن عمليات البث المباشر العابر للحدود في ضوء احكام القانون الدولي، رسالة دكتوراه، جامعة المنوفيه ، كلية الحقوق، 2008.

20. أخيكاني، ميري كاظم ، خدمات البث الفضائي ، دراسة مقارنة بين القانون العراقي وقانون الاتحاد الاوروبي، مجلة القادسية ، كلية

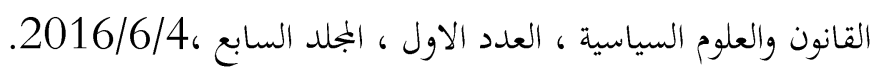

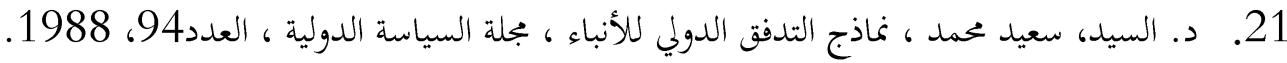

22. د. الفار، عبد الواحد ، حرية الرأي والفكر والعقيدة في المواثيق العالمية ، مجلة الدراسات الاعلامية، العدد80،

23. د. عبد المتعال، صلاح ، الآثار الاجتماعية للتنمية الثقافية والاتصال الاعلامي بواجهة حضارية ، ورقة مقدمة الى وقائع مؤتمر قضية

الثقافة والاعلام في مصر، 1994.

24. الاعلان العالمي لحقوق الانسان-CIA، تاريخ الزيارة 2019/8/21،

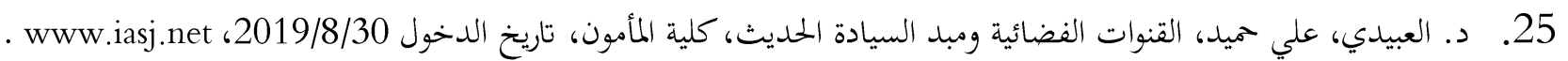
26. د. العمر، ناصر بن سليمان، البث المباشر، حقائق وارقام، تاريخ الزيارة 2019/8/21، https:ar.islamway.net.book.

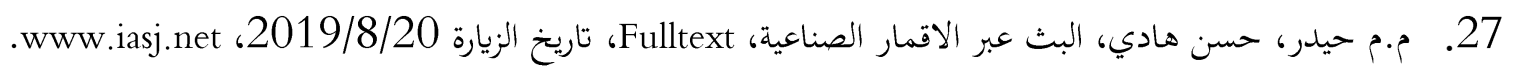

\section{الثهوامش}

$$
\text { (1) د. بسيوني، الحماية الدولية للأقمار الصناعية في ضوء قواعد القانون الدولي العام: ص325. }
$$

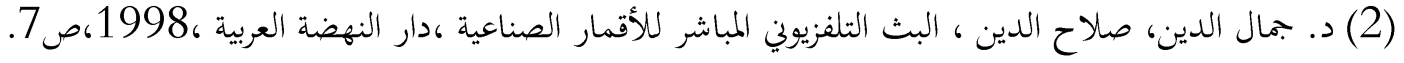

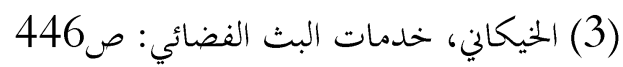

(4) ينظر : عثمان، المسؤولية الدولية عن عمليات البث المباشر العابر للحدود في ضوء احكام القانون الدولي: ص20.

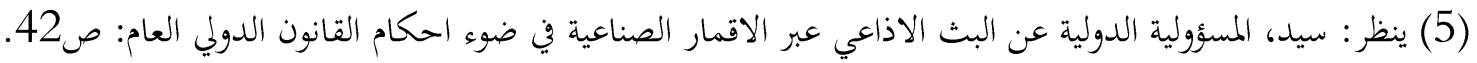

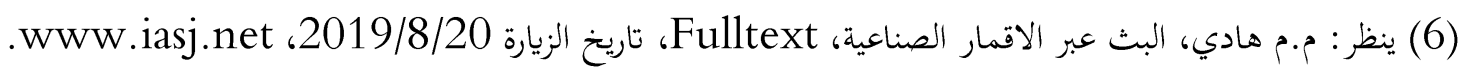
(7) ينظر: لطفي، البث الاذاعي عبر التوابع الصناعية وحقوق المؤلف: ص11

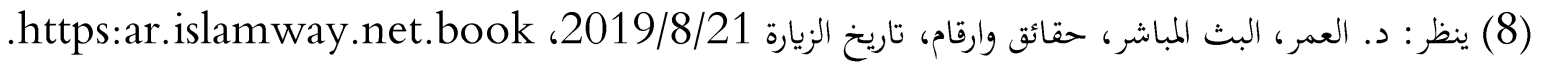

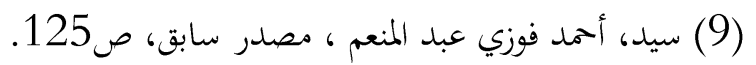
(10) بجموعة مؤلفين، حرية تداول المعلومات، دراسة قانونية مقارنة، مؤسسة حرية الفكر والتعبير، القاهرة،11، 11، 2011، ص18. Library.https//www.cia.cov 2019/8/21 الاعلان العالمي لحقوق الانسان-CIA، تاريخ الزيارة

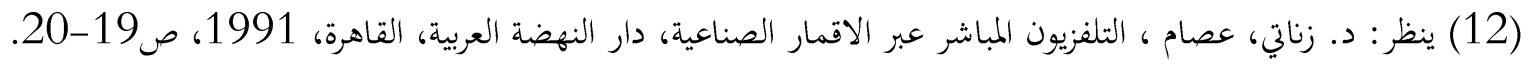


(13) ينظر : عثمان، جمال عبد الفتاح ، مصدر سابق ص61-62.62.

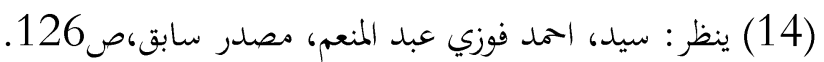

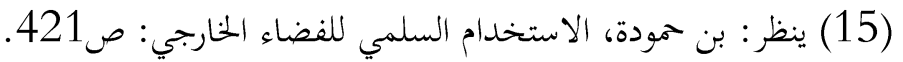

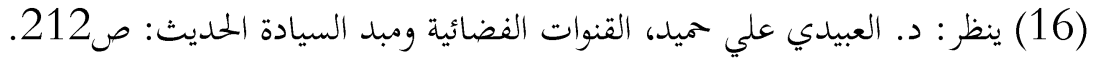

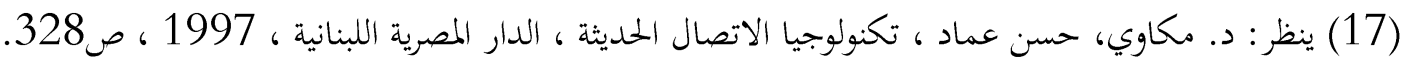

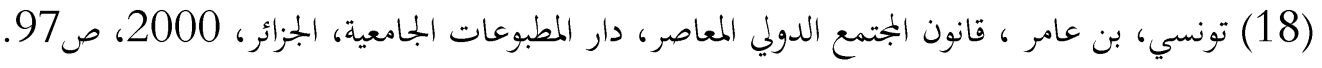

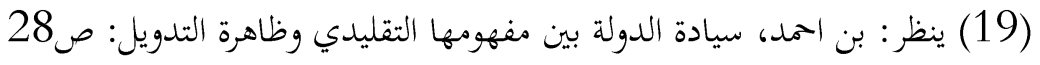

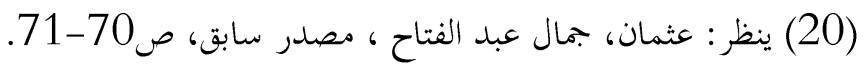

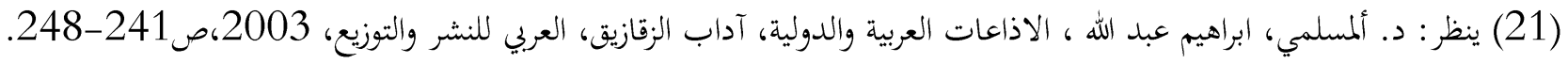

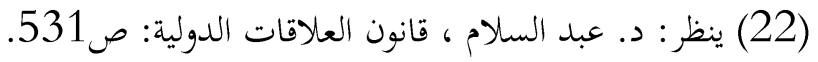

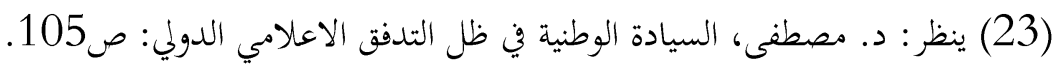

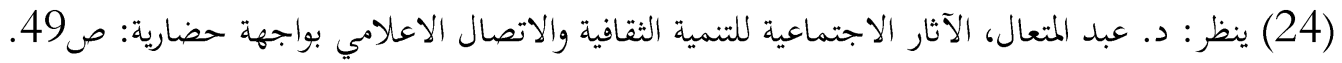

(25) ينظر : د. العبيدي، علي مميد ، مصدر سابق، ص213.

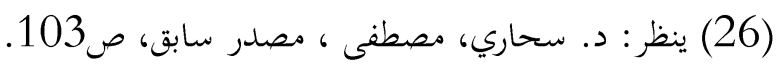

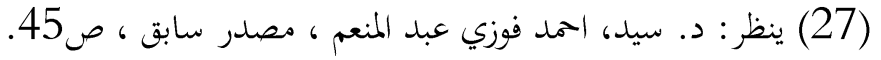

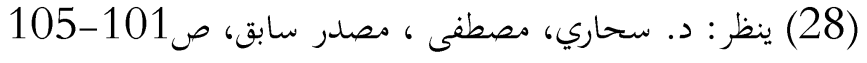

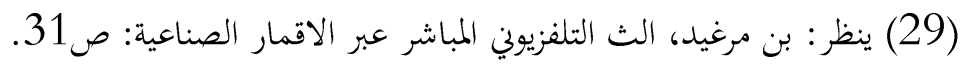

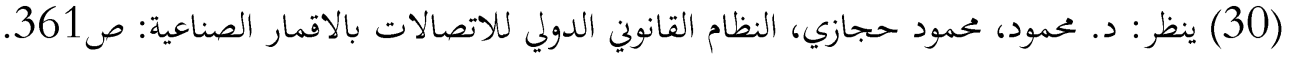

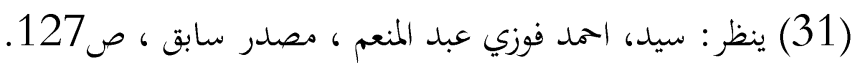

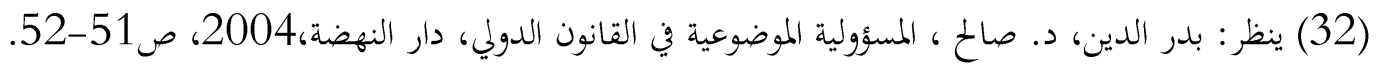

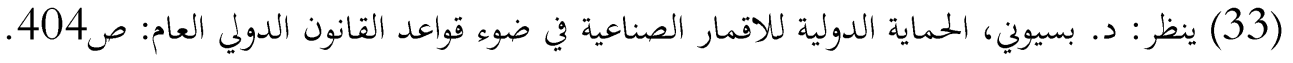

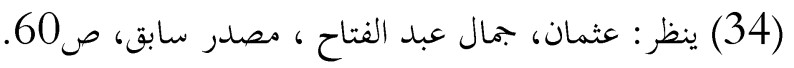

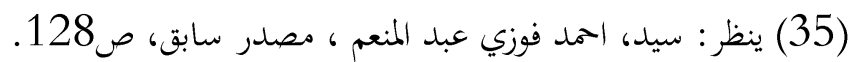

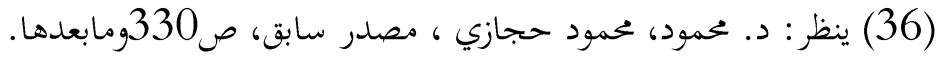

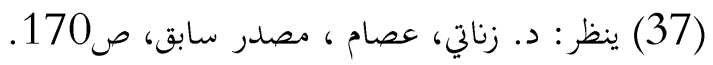

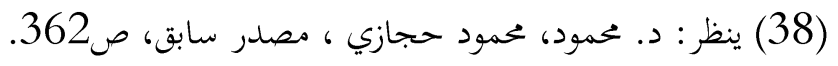

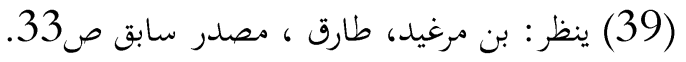

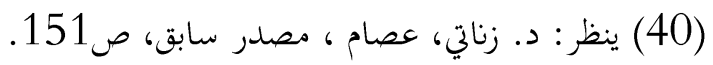

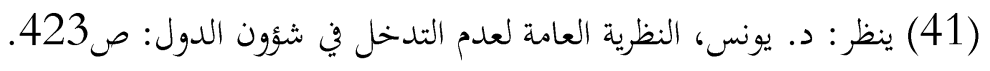




$$
\begin{aligned}
& \text { (42) ينظر: بن مرغيد، طارق ، المصدر نفسه، ص33-34. }
\end{aligned}
$$

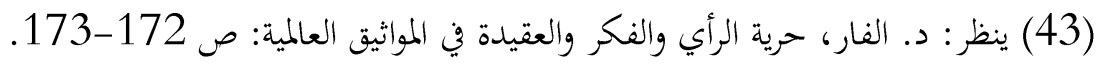

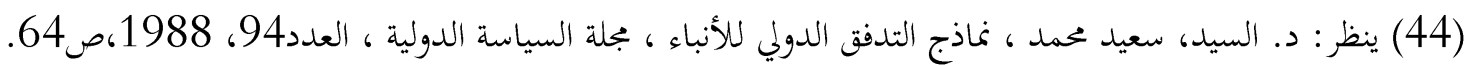

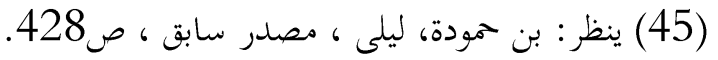

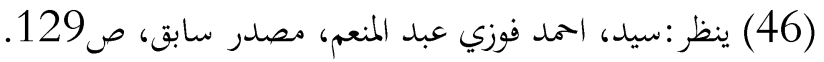

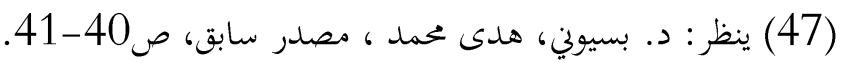

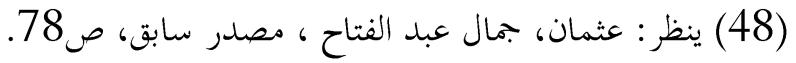

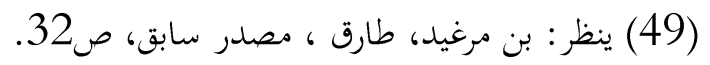

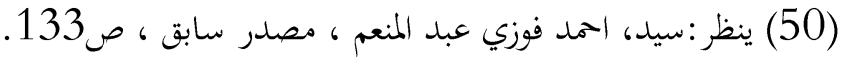

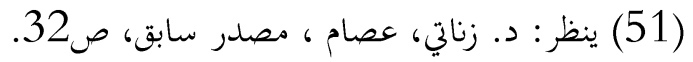

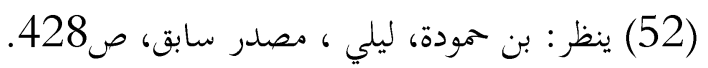

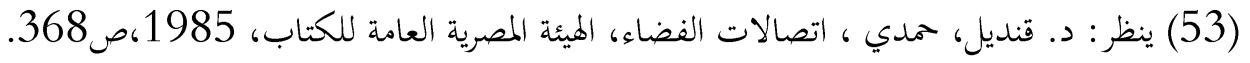

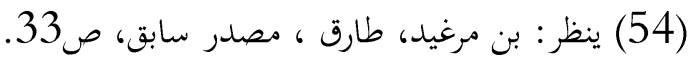

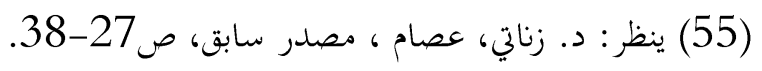

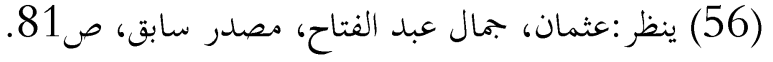

